\title{
3D Confocal Scanning Laser Microscopy to Quantify Contact Angles in Natural Oil-Water Mixtures
}

\author{
A. Salim ${ }^{1 *}$, J. Sausse ${ }^{2}$, J. Pironon ${ }^{2}$, M. Fourar ${ }^{3}$ and C. Le Carlier De Veslud ${ }^{4}$ \\ 1 Institut de Mécanique des Fluides de Toulouse, UMR 5502 CNRS/INP/UPS, 2 avenue Camille Soula, 31400 Toulouse - France \\ 2 UMR 7566, Géologie et Gestion des Ressources Minérales et Énergétiques, \\ UHP Nancy-1, BP 239, 54506 Vandoeuvre-lès-Nancy Cedex - France \\ 3 École des Mines de Nancy - LEMTA, Parc de Saurupt, 54042 Nancy Cedex - France \\ 4 CRPG-CNRS et LIAD-ENSG, 15 rue Notre-Dame-des-Pauvres, 54501 Vandøeuvre-lès-Nancy - France \\ e-mail: abdelkader.salim@imft.fr - judith.sausse@g2r.uhp-nancy.fr - jacques.pironon@g2r.uhp-nancy.fr \\ Mostafa.Fourar@ensem.inpl-nancy.fr - carlier@crpg.cnrs-nancy.fr
}

\begin{abstract}
Résumé - Microscopie confocale à balayage laser 3D pour quantifier les angles de contact des mélanges naturels diphasiques eau-huile - La mesure de l'angle de contact est l'un des moyens les plus utilisés pour quantifier la mouillabilité et l'énergie de surface d'un matériau en contact avec un/ou plusieurs liquides. Ce travail présente une méthode basée sur le principe du microscope confocal à balayage laser pour mesurer l'angle de contact d'une goutte déposée sur une surface solide. Plusieurs systèmes solide-fluide1-fluide2 ont été étudiés. La méthode CSLM est basée sur l'acquisition d'une série d'images en $2 \mathrm{D}$ et suivant l'axe $Z$ d'une goutte fluorescente. La reconstruction de l'image en 3D d'une goutte est obtenue par la somme des images $2 \mathrm{D}$ à différents niveaux $Z$ de la surface solide. Les résultats obtenus sont comparés à ceux obtenus par goniométrie, méthode de mesure conventionnelle de l'angle de contact. Ces deux méthodes sont en accord pour les différents systèmes solide-liquide-air étudiés. La modélisation géométrique en 3D de la goutte, obtenue à partir des images CSLM, permet cependant d'étudier la distribution locale des angles de contact en périphérie des gouttes et de mieux détecter l'influence des hétérogénéités locales d'une surface solide sur la valeur finale de l'angle de contact solide-liquide-gaz.
\end{abstract}

\begin{abstract}
D Confocal Scanning Laser Microscopy to Quantify Contact Angles in Natural Oil-Water Mixtures - The contact angle of a liquid drop on a solid surface is one of the most simple, useful and sensitive parameters in the hydraulic sciences to quantify and to qualify the wettability and the surface energy of different materials. In this paper, a confocal scanning laser microscope (CSLM) has been used to quantify contact angle and then the wettability. This technique uses a laser scan and allows series of 2-D images of a fluorescent liquid droplet set on various solid surfaces to be recorded. The generation of 3-D images is carried out with the summation of several images acquired with a regular step along the CSLM z-axis. The results obtained are compared and discussed with those obtained with a conventional goniometric technique for different solid-liquid-air systems. CSLM results show that similar values are obtained with both methods. Thus, this technique shows that the length interval of sampling between the solid contact and the curvature rupture of the drop seems to be essential to correctly estimate the contact angles. This method allows the study of the local contact angle along the periphery of the drop, and the deformation of the drop shape. Construction of 3-D images shows that drops are characterized by complex morphologies and that the local contact angles can be modified by chemical heterogeneities in the fluids.
\end{abstract}




\section{INTRODUCTION}

The concept of contact angle has been widely used in studies involving characterization of solid-fluid1-fluid2 interfacial interactions. When a liquid droplet is placed on a solid surface, the droplet profile (i.e. a vertical section of the droplet perpendicular to the solid surface) is controlled by the balance between the liquid-gas $\left(\gamma_{L}\right)$, the solid-gas $\left(\gamma_{S}\right)$ and the solid-liquid $\left(\gamma_{S L}\right)$ interfacial tensions. A liquid characterized by a low surface tension set on a smooth solid with a high surface energy produces a small contact angle $(\theta)$ between the two phases. When $\theta$ is equal to zero, the liquid spreads along the solid and the droplet becomes a liquid film. On the contrary, when the liquid is characterized by higher surface tension and the solid by lower surface energy, the contact angle becomes nonzero, even larger than $90^{\circ}$, at which the liquid on the solid surface is considered as non-wetting.

Contact angle measurement is a key parameter to determine the solid wettability which characterizes the tendency of a fluid to spread along a solid surface in the presence of another (Crocker and Marchin, 1988). The wettability has a crucial impact on flow during oil recovery and upon the volume and distribution of the residual oil (Craig, 1971; Samathiel, 1973; Morrow et al., 1986; Anderson, 1987; Morrow, 1991; Jadhunandan and Morrow, 1995).

The main methods to measure the contact angle are based on optical observations on the scale of a hundred micrometers. A conventional method to measure the contact angle of a liquid sessile droplet on a smooth solid surface is to observe a droplet section through an optical microscope (Leger and Joanny, 1992). Then, a photograph of the droplet profile is taken. This picture shows therefore a more or less complex profile and the droplet tangent line is then drawn from the three phase contact point. A direct measurement of the angle between the tangent line and the solid section is then performed. However, the three phase contact point can be difficult to determine if, for example, the photograph is poorly numerically defined or badly exposed. Moreover, a precise quantification of the contact angle is directly linked to the graphical positioning of this particular point.

Another method called the " $\theta / 2$ method" (Yang and Lin, 2003) assumes that the sessile droplet is a part of a perfect sphere. The contact angle is calculated as the angle defined by two lines, the line between the three phase contact point and the sphere apex and the solid section. Only the height of the droplet and the contact diameter are needed. However, when the droplet reaches a significant volume, gravity effects can influence and modify its spherical shape and lead to errors in the $\theta$ value determination. Indeed, some recent studies show the effect of droplet size on the determination of contact angles for different solid-liquid-air systems (Li, 1996; $\mathrm{Gu}, 2001)$. The contact angle decreases when the base radius of the liquid droplet increases. For high Bound number (i.e. large volume or small surface tensions), the spherical assumption causes errors for the contact angle measurements. Yan and Lin (2003) proposed some corrections of the measurement error using the mean difference between contact angles obtained with a $\theta / 2$ method and a theoretical calculation. Nevertheless, the $\theta / 2$ method can be used in the case of small droplet volume.

Zhang and Chao (2002) used a laser shadowgraphy method to measure dynamic contact angles and the spreading rate of liquid sessile droplets on a non-transparent metal substrate. Based on geometrical optics, the dynamic contact angle of the droplet is determined with the measurements of the droplet contact and the refracted-shadowgraphic-image diameters.

Whatever the methodology used, the contact angle of a solid-fluid1-fluid2 system is not unique but fluctuates between two extreme values denoted $\theta_{r}$ and $\theta_{a}=\theta_{r}+\Delta \theta$ respectively named the receding and the advancing angle. The hysteresis $\Delta \theta$ is mainly linked to the solid surface roughness and its heterogeneity. In this paper, a new microscopic approach to contact angle measurement is proposed. It uses Confocal Scanning Laser Microscopy (CSLM) to obtain 3D views of liquid droplet positioned on solid material plates. This microscopic technique requires only small liquid droplet with a volume lower than $1 \mathrm{~mm}^{3}$ and thus avoids gravity effects. It allows measurement at low resolution $(<1 \mu \mathrm{m})$ and it is very sensitive to chemical heterogeneities. This technique has been previously used for 3D reconstruction of petroleum fluid inclusions in minerals (Pironon et al., 1998).

\section{MATERIALS AND METHODS}

Two main methods are used to quantify the contact angles of the different phase systems. First, each contact angle is quantified with a 2D image obtained by goniometry (LERMAB laboratory, University of Nancy, France). In a second step, sequences of 2D images are obtained by CSLM ( $G 2 R$ laboratory, University of Nancy, France) and 3D models of the liquid droplets are constructed with the gOcad 3D modeler (Earth Decision Sciences ${ }^{\mathrm{TM}}$, Paradigm ${ }^{\mathrm{TM}}$; Mallet, 2002). Finally, a statistical study of the 3D angles is performed and values are compared to those obtained with goniometry.

\subsection{Goniometry}

The goniometer uses a white light source to illuminate the drop samples. A sighting telescope connected to a digital camera which produces a sharply defined image of the sessile drops which are observed as silhouettes (Fig. 1). The contact angle is determined from the resulting $2 \mathrm{D}$ numerical images using the $\theta / 2$ method. The droplets are small enough to define spherical shapes. 


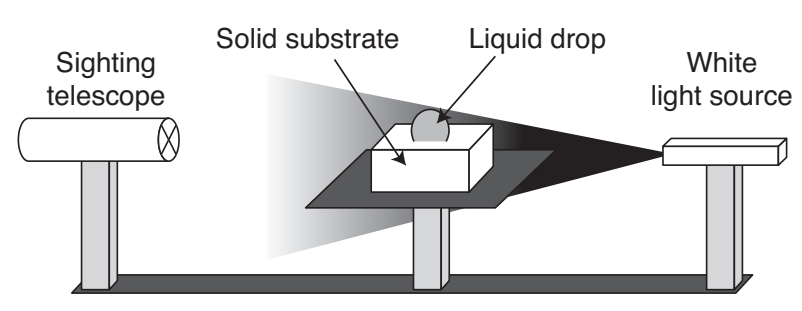

Figure 1

Schematic view of the conventional goniometry.

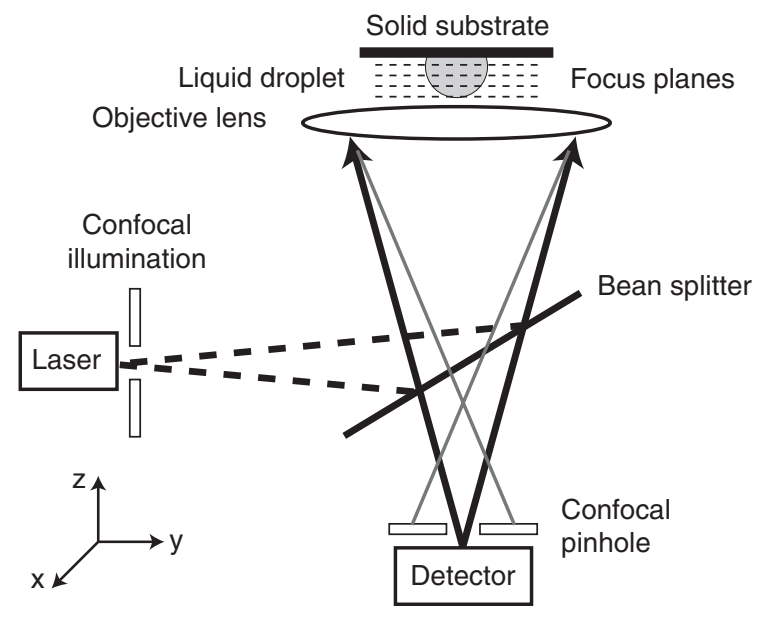

Figure 2

Schematic view of the optical path during confocal recording of the image planes of a droplet.

\subsection{Confocal Scanning Laser Microscopy}

The confocal principle is outlined in Figure 2. CSLM is based on the filtering of emission light (fluorescence) by a confocal pinhole. The confocal pinhole selects the light emitted by the sample (a liquid fluorescent droplet) in the plane of the focused incident laser spot. Out-of-focus fluorescence is imaged before or after the confocal pinhole and cannot reach the detector. A 2-D image of a volume of the droplet centered around the focal plane (referred to as an optical section) is generated by performing a raster sweep of the droplet in that focal plane. As the laser scans across the specimen, the analog light signal is detected by the photo-multiplier and converted into a digital signal, contributing to a pixel-based image displayed on a computer monitor attached to the CSLM. The relative intensity of the fluorescent light, emitted from the laser hit point, corresponds to the intensity of the resulting pixel in the image. The plane of focus (Z-plane) is selected by a computer-controlled fine-stepping motor which moves the microscope stage or the objectives up and down. Typical focus motors can adjust the focal plane in as little as 0.1 micron increments. A 3-D reconstruction of a specimen can be generated by stacking 2-D optical sections collected in series (Fig. 2). The objective used for high resolution CSLM acquisition is a Nikon objective apoplan $60 \times$ with a numerical aperture of 1.40 working in oil immersion. Images have been acquired at G2R laboratory (Nancy, France) on a Biorad Rainbow system and at IBMP (Strasbourg, France) on a Zeiss LSM510 system.

\subsubsection{D CSLM Images Reconstructed along XZ and YZ Planes}

On each $X Z$ and/or $Y Z$ section the droplet profile tangents are drawn from the three phase contact point between the solid, air, and liquid phases. The three phase contact point appears clearly on the CSLM pictures as the difference of the fluorescence intensities between a fluorescent oil and nonfluorescent phase (water or air) and the solid surface.

As shown in Figure 3, a misestimation of the droplet median plane position by visual determination can generate some variations of the contact angle values. One theoretical example is proposed and illustrated in Figure 4. Series of angles are calculated for several $Y Z$ section photographs, around the exact median plane of a perfect half sphere

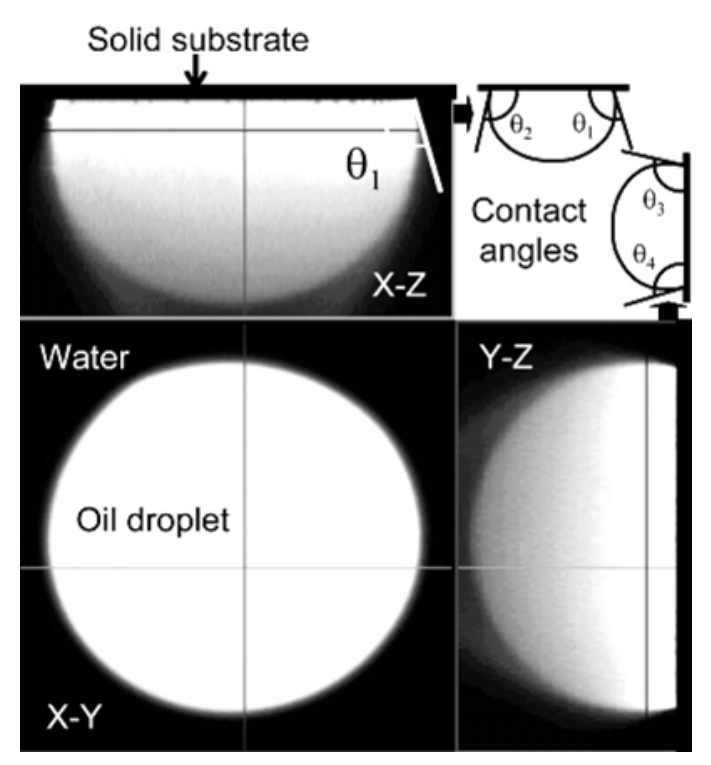

Figure 3

$X-Y, X-Z$ and $Y-Z$ sections of an oil droplet in water set on a glass surface and obtained by Confocal Scanning Laser Microscopy. The different contact angles are obtained with different $Y Z$ and $X Z$ sections of the droplet. 
corresponding to the liquid droplet. Contact angles for each section along $Y Z$ planes (for different $X_{i}$ positions) vary from 42 to $83^{\circ}$. The angle decreases when the $X_{i}$ position is farfrom the median plane. Accurate values of contact angles $\left(\right.$ error $\left.<1^{\circ}\right)$ can be achieved for $X_{i}$ positions of $\pm 15 \%$ of droplet diameter from the median plane. Consequently, the uncertainty which can be generated by the median plane location can be neglected.

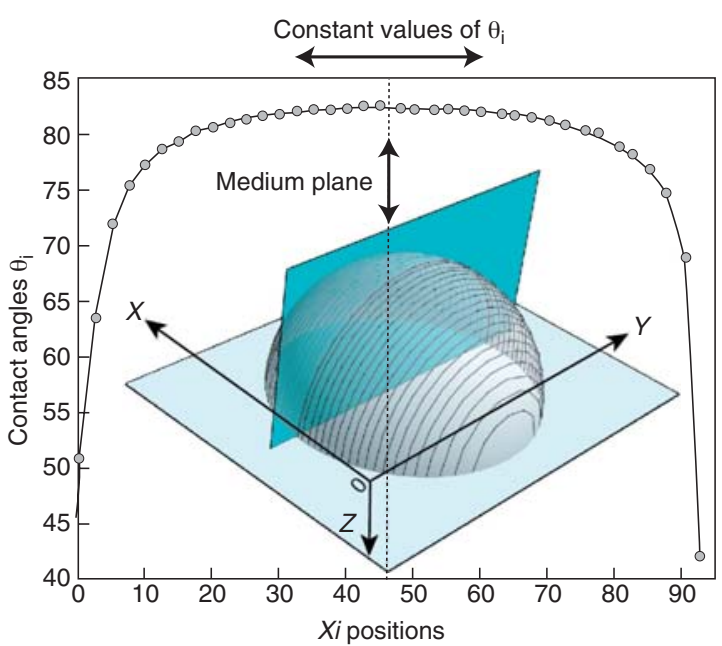

Figure 4

$\theta$ variations observed for a near perfect half of sphere set a solid plane. Different sections are realized for different $X_{i}$ positions and the respective contact angles $\theta_{i}$ are calculated A threshold is observed with a constancy of $\theta_{i}$ values (errors $<1^{\circ}$ ) for $X_{i}$ position of $\pm 15 \%$ of droplet diameter from the medium plane.

\subsubsection{IMAGE 3D CSLM}

A first treatment of CSLM pictures is realized before the droplet reconstruction with gOcad. A systematical and identical initial image processing is done to transform the CSLM million colors or RVB picture in a binary color picture. Since the external edges of the droplet are of interest for the contact angle determination, the objective of this image processing is to delete the inner zone of the droplet image to obtain a precise outer edge characterized by black pixels (Fig. 5).

The image processing is performed in several steps described in Figure 5. The initial CSLM image is a bitmap image characterized by a "million colors" color chart. Fluorescent zones (oil) appear with various levels of green (Fig. 5A). Another type of colour palette is used to better contrast non- fluorescent and fluorescent zones (Fig. 5B). Then, a first threshold is applied to define the outer edges of the oil droplet (Fig. 5C). Edges are enhanced and the image is simplified with a binary black and white colour palette (Fig. 5D).

Resulting black and white pictures are loaded in a Visual Basic code to transform the black pixel positions into ASCII data in which each fluorescent pixel of the outer edges of the droplet, assuming that this edge is characteristic of the oil-air interface, is characterized by a $X Y$ and $Z$ coordinates (Figs. $5 E, F)$. The $Z$ coordinate is constant for each section and corresponds to the height level at which the CSLM picture was obtained.

Ten or more sections are treated for each droplet and a database is obtained for different samples (Table 1).

The gOcad model allows the modeling of realistic and complex natural objects thanks to a variety of preprocessed discrete objects as pointset, polygonal lines, triangulated surfaces or tetrahedral volumes (Mallet, 2002). The first type of object corresponds to the 3D mapping of discrete $X Y Z$ data in the camera window of gOcad. This object is the basis of the

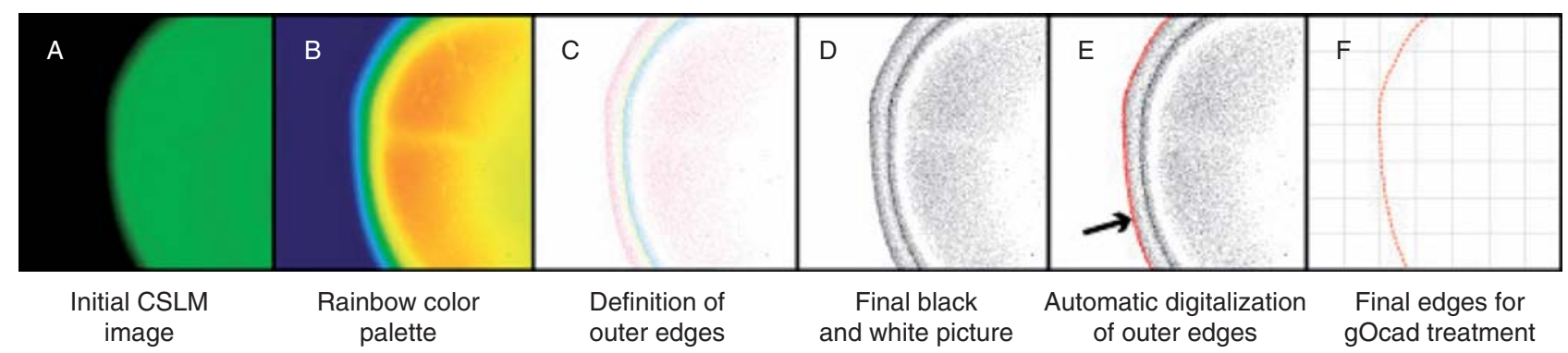

Figure 5

Different steps of the CSLM image analysis. (A) initial CSLM image and various green fluorescent zones (oil). (B) Different colour palette to better contrast non- fluorescent and fluorescent zones. (C) First thresholding to define the outer edges of the oil droplet. (D) Edge enhancement and resulting binary black and white image. (E) Automatic digitization of the droplet outer edge. (F) $X Y$ coordinates of the droplet edge. These data are used to build the gOcad model. 
TABLE 1

Different solid-fluid1-fluid2 systems used in this study. Only air is used as fluid1, different natural oils or water characterizes the fluid2. Small amounts of fluorochromes are added to water to produce fluorescence. The types of objective and the laser parameters used during the CSLM acquisition are also indicated Salim et al., A 3D laser microscopy to quantify contact angles

\begin{tabular}{|c|c|c|c|}
\hline SOLID & FLUID1 & FLUID2 & CSLM \\
\hline Quartz & Air & Oil H9 & $\begin{array}{c}\text { Objective } * 40 \text { No immersion oil } \\
\text { wavelength laser used } 633 \mathrm{~nm}\end{array}$ \\
\hline Vermiculite & Air & Oil H9 & $\begin{array}{l}\text { Objective } * 40 \text { No immersion oil } \\
\text { wavelength laser used } 633 \mathrm{~nm}\end{array}$ \\
\hline Graphite & Air & $\begin{array}{c}\text { Water + fluorochromes } \\
\text { Oil H5 } \\
\text { Oil H9 } \\
\text { Oil H14 }\end{array}$ & $\begin{array}{l}\text { Objective * } 10 \text { No immersion oil } \\
\text { wavelength laser used } 514 \mathrm{~nm}\end{array}$ \\
\hline Paraffin & Air & $\begin{array}{c}\text { Water + fluorochromes } \\
\text { Oil H5 } \\
\text { Oil H9 } \\
\text { Oil H14 }\end{array}$ & $\begin{array}{l}\text { Objective * } 10 \text { No immersion oil } \\
\text { wavelength laser used } 514 \mathrm{~nm}\end{array}$ \\
\hline Glass & Air & $\begin{array}{c}\text { Oil H5 } \\
\text { Oil H9 } \\
\text { Oil H14 }\end{array}$ & $\begin{array}{l}\text { Objective * } 40 \text { No immersion oil } \\
\text { wavelength laser used } 633 \mathrm{~nm}\end{array}$ \\
\hline Stibnite & Air & $\begin{array}{c}\text { Water + fluorochromes } \\
\text { Oil H5 } \\
\text { Oil H9 } \\
\text { Oil H14 }\end{array}$ & $\begin{array}{l}\text { Objective }^{*} 10 \text { No immersion oil } \\
\text { wavelength laser used } 514 \mathrm{~nm}\end{array}$ \\
\hline
\end{tabular}

model construction. Here, the different CSLM pictures are numerically treated to obtain black and white pictures and $X Y Z$ points correspond to the $X Y Z$ positions of the black pixels which are the initial fluorescent zones and edges of the oil droplet (Fig. 5). Each pointset object represents one CSLM image i.e. one specific height level of $Z$. For each sample, more than 15 sections and initial CSLM pictures are taken which represent droplet height samplings from $24.5 \mu \mathrm{m}$ (stack sizes for the glass-oil-air system) to $170 \mu \mathrm{m}$ (stack sizes for the paraffin-oil-water system). The differences of height samplings are due to the wettability characteristics of the solids and the more or less intense spreading of the oil droplets on the solids.

In a second stage, a polygonal line is fitted to each initial pointset. These lines are densified with a minimum segment length corresponding to a sampling of around $5^{\circ}$ along the droplet perimeter.

Finally, a triangulated mesh on the surface is built from the set of lines obtained in the second stage. This mesh is densified and fitted to data by using the DSI method (Mallet, 1992, 2002; Fig. 6). All the process from the image treatment to the 3D surface construction constitutes a robust design and was applied identically to all the samples presented in this paper.

The complex surfaces can be characterized by a 3D property which attributes $n_{x}, n_{y}$ and $n_{z}$ coordinates to all the normals of the triangles constituting the surface (Fig. 6). Only the contact zone has been characterized by these normals (i.e. the droplet first curvature). This contact zone corresponds generally to a height of around 25 microns from the solid plates. The contact angles are deduced from a trigonometric relation using the $n_{z}$ coordinates of the normal unit vectors (Equation 1, Fig. 7).

$$
\theta=\frac{\pi}{2}+\operatorname{Arcsin}\left(n_{z}\right)
$$

where $\theta$ is the contact angle and $n_{z}$, the $Z$ coordinate of the normal vector.

The gOcad modeler calculates the normals of the whole surface in the contact zone allowing the whole contact angles on the entire perimeter of the oil droplet to be systematically defined. The different values of $\theta$ are then treated statistically.

\section{SAMPLING}

Each sample is represented by a small droplet of liquid set on a solid surface. The droplets diameter is less than $2 \mathrm{~mm}$ in diameter in each case. The liquid is in equilibrium with its vapor. Three natural fossil dead oils have been used for the 


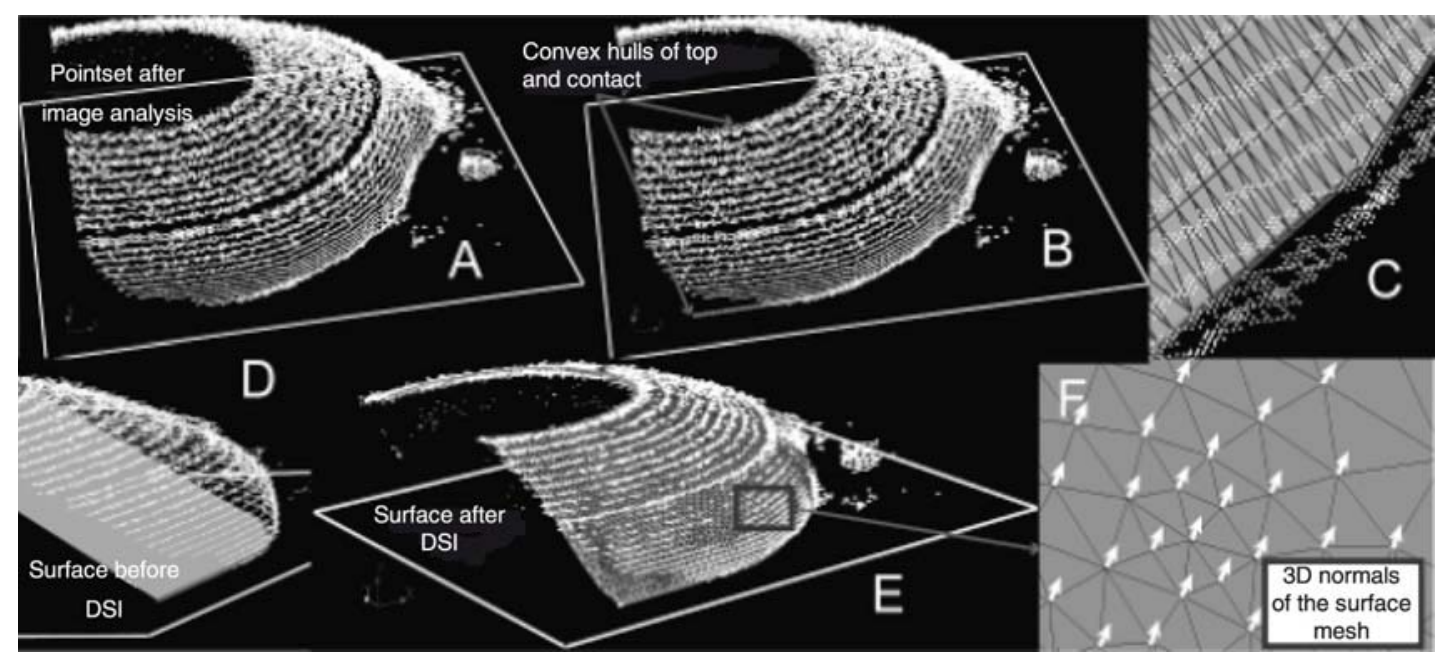

Figure 6

Different steps of the gOcad model building. (A) The initial CSLM image is imported into gOcad by the way of a XYZ pointset (white discrete dots). (B) The different convex hulls of each $Z$ level are built, here the top and bottom curves are shown. These curves correspond to the future skeleton of the surface and each level of the curve points corresponds to the initial sampling of the CSLM image. (C) A regular triangulation is first used to build the surface. (D) This surface is initially fit to the different curves without any interpolation with the Discrete Smooth Interpolation mode (DSI) and the triangulated mesh does not fit the original pointset. (E) With some geometric constraints on the surface object and on the initial points, the final surface is irregularly meshed to fit the points and finally obtained with a few DSI iterations. (F) The surface corresponds to the CSLM points. The XYZ coordinates of the normals to each triangle in the vicinity of the contact zone are then extracted and exported in a text file. The contact angles are calculated with these coordinates.

experiments (oil5, oil9 and oil14). They contain aromatic molecules, more or less complex, that are mainly responsible for fluorescence emission under laser excitation. The chemistry of these molecules is not perfectly known. Graphite, quartz, glass, paraffin, stibnite and vermiculite are used as smooth solid substrates. Two types of samples are used to estimate the contact angles with the CSLM technique. In the case of solid-liquid-air systems, a liquid droplet is set on a solid surface with a micro-syringe. After each measurement, ethanol is used to perfectly clean the solid surface in order to obtain reproducible results. Cleaning of traces of organic contaminants on mineral surfaces is first obtained by washing with dichloromethane followed by rinsing with ethanol in ultrasonic tank. The presence of residual precipitated salts is eliminated by rinsing with pure water, followed by heating at $60^{\circ} \mathrm{C}$ during 1 hour. After this procedure, tests of wetting angle measurement reproducibility were performed and the results show a reproducibility range lesser than $2^{\circ}$. In the case of solid-liquid-water systems, the liquid droplet (Oils 5, 9 or 14) is set on a solid surface and then immersed in an immiscible liquid (water, Table 1). The two liquids are then retained firmly between two glass wafers thereby avoiding any perturbations of the droplet shape during the measurement.

The different systems used in this study and the type of objective are detailed in Table 1.

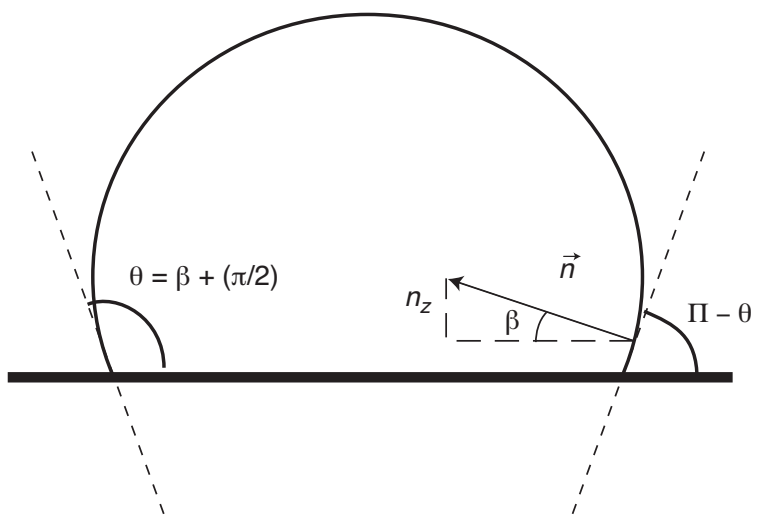

Figure 7

Schematic view of the trigonometric relations existing between the droplet surface and the contact angle $\theta$.

\section{RESULTS}

Contact angles measured with the $X Z$ or $Y Z$ CSLM image analysis and those measured by the $\theta / 2$ method for different solid-liquid-air systems are presented in Table 2 and Figure 8 with their mean values of contact angles weighted by their standard deviations. 
TABLE 2

Mean contact angles weighted by their standard deviations obtained for the different systems with the two first techniques: the Matlab graphical image analysis using the median CSLM sections and the Goniometry method Salim et al., A 3D laser microscopy to quantify contact angles

\begin{tabular}{|c|c|c|c|c|}
\hline SOLID & FLUID1 & FLUID2 & 2D CSLM & GONIOMETER \\
\hline Quartz & Air & Oil $\mathrm{H}_{9}$ & $7.26 \pm 0.59$ & 9 \\
\hline Vermiculite & Air & Oil $\mathrm{H}_{9}$ & $8.9 \pm 0.76$ & 8 \\
\hline Graphite & Air & Water + fluorochromes & $75 \pm 0.97$ & 82 \\
\hline Graphite & Air & Oil $\mathrm{H}_{5}$ & $25.4 \pm 0.84$ & 20 \\
\hline Graphite & Air & Oil $\mathrm{H}_{9}$ & $11.4 \pm 0.52$ & 15 \\
\hline Graphite & Air & Oil $\mathrm{H}_{14}$ & $7.86 \pm 0.72$ & 4 \\
\hline Paraffin & Air & Water + fluorochromes & $69.75 \pm 0.7$ & 100 \\
\hline Paraffin & Air & Oil $\mathrm{H}_{5}$ & $26 \pm 1.33$ & 20 \\
\hline Paraffin & Air & Oil $\mathrm{H}_{9}$ & $21.7 \pm 0.86$ & 18 \\
\hline Paraffin & Air & Oil $\mathrm{H}_{14}$ & $10.4 \pm 0.59$ & 7 \\
\hline Glass & Air & Oil $\mathrm{H}_{5}$ & $6.7 \pm 0.9$ & 4 \\
\hline Glass & Air & Oil $\mathrm{H}_{9}$ & $13.9 \pm 1.27$ & 26 \\
\hline Glass & Air & Oil $\mathrm{H}_{14}$ & $7.8 \pm 0.46$ & 0 \\
\hline Stibnite & Air & Water + fluorochromes & $53 \pm 1.26$ & 65 \\
\hline Stibnite & Air & Oil $\mathrm{H}_{5}$ & $21.5 \pm 1.06$ & 27 \\
\hline Stibnite & Air & Oil $\mathrm{H}_{9}$ & $11.6 \pm 0.29$ & 10 \\
\hline Stibnite & Air & Oil $\mathrm{H}_{14}$ & $7.1 \pm 0.57$ & 4 \\
\hline
\end{tabular}

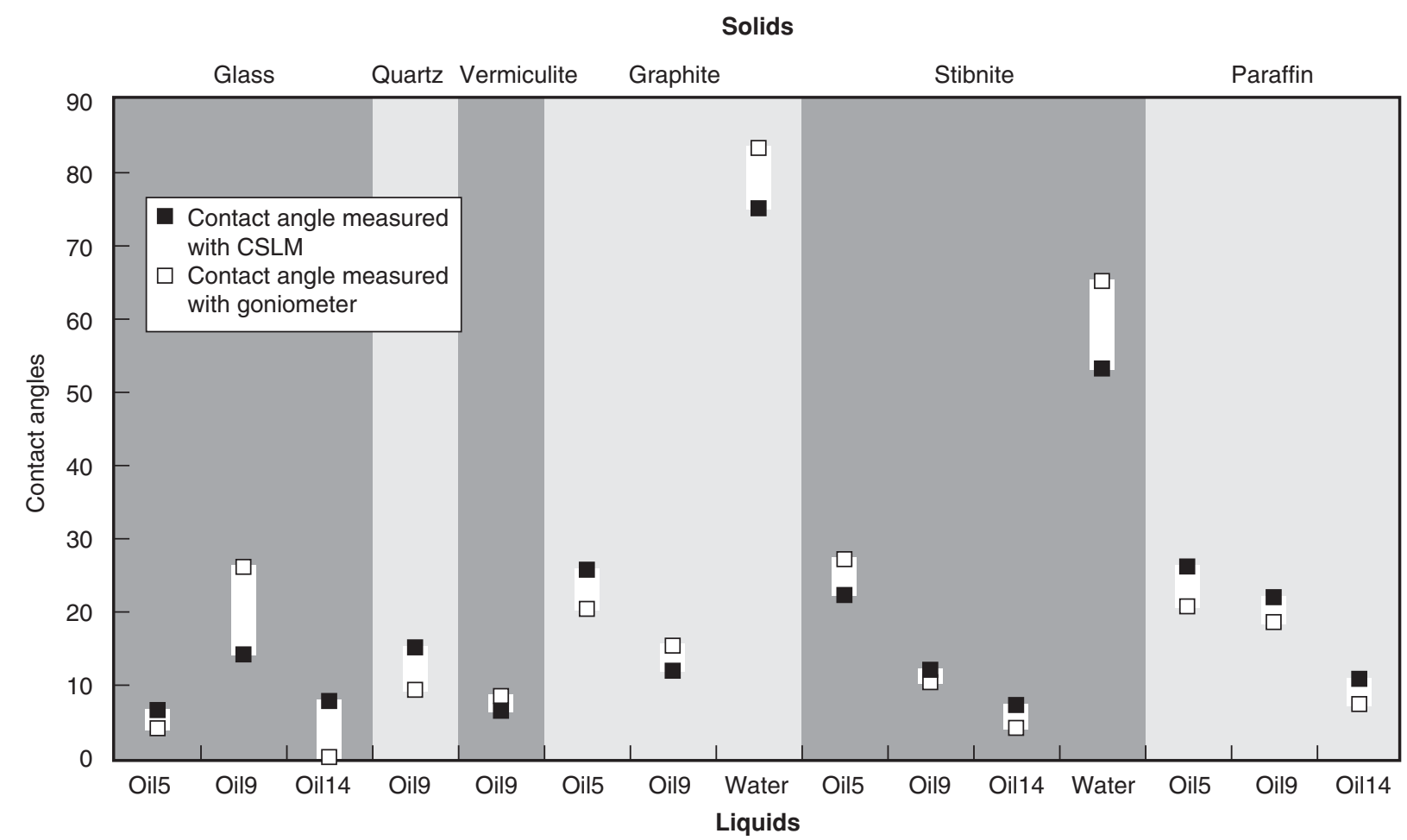

Figure 8

Values of contact angles measured by CSLM method compared with those measured by the goniometry method for different specimens of solid-liquid-air. 
Figure 8 shows that there is good agreement between goniometer data and image analysis from 2D CSLM pictures. The same orders of magnitude are obtained for the various contact angles which define correctly the classical wettability of the different solid-liquid-air systems and therefore show the validity of the CSLM method.

However, some systems present slightly different values of $\theta$ angles with the two techniques: glass-oil9-air, glass-oil14-air, graphite-water-air and stibnite-water-air (Fig. 8). In these cases, the CSLM methodology gives contact angle of $\pm 10^{\circ}$ in comparison with the classical goniometric technique. This discrepancy can be explained by the observation scale which is different for the two techniques. The location of the three phase contact point is crucial to precisely define the correct profile tangent of the droplet and the resulting contact angle. The contact angle is generally used in the interpretation of storage capacities or permeability of petroleum reservoirs or aquifers. Afterward, a precise value of $\theta$ is required.

In order to improve the approximations due to $Z$ plane selection and tangent drawing, a gOcad statistical treatment has been developed from the 3D droplet construction. All the contact angles deduced from the difference surface normals (Fig. 6) are presented in Figure 9. In this plot, the frequency histograms are determined for the contact angle distributions of the different three phase systems. A first observation shows that no real unimodal distribution exists and that $\theta$ values are highly variable for each system. From the contact points $(Z=0)$ to the maximal $Z$ samplings, variations of more than $20^{\circ}$ can be observed for all the three phase systems and these variations are nearly continuous from $0^{\circ}$ to $40^{\circ}$ for the glassoil9-air system. These variations result from the $3 \mathrm{D}$ surface construction of the droplet which produces complex morphologies due to the image treatment and the evolution of the fluorescence intensity with $Z$ during CSLM acquisition. Despite this variability, some easily recognizable trends are observed and describe the main modes for contact angles. Indeed, the quartz-oil9-air contact angles are well distributed with two modes at $6^{\circ}$ and $8^{\circ}$ but a mean value of $6.5^{\circ}$ and a standard deviation of $1.7^{\circ}$ ( $9^{\circ}$ for the goniometer measurements). The same observation can be done for the graphiteoil9-air system with again two modes at $8^{\circ}$ and $14.5^{\circ}$. The goniometer value of $15^{\circ}$ fits with the second mode obtained with CSLM technique. A similar relation is more or less reproducible for all investigated systems: the goniometer angle is similar to the second mode deduced from CSLM technique.

It implies that the two different scales of observations can induce non-negligible differences in the determination of contact angles. The higher the angle value, the higher this difference becomes. Glass and paraffin solids are badly described by goniometer values with respectively $26^{\circ}$ and $18^{\circ}$ for goniometer and $15.0^{\circ} \pm 6.5^{\circ}$ and $15.7^{\circ} \pm 3.3^{\circ}$ for CSLM angle values but highly variable distributions and different modal values. The lower the contact angle, the higher the zoom used by goniometry is and therefore the closer the scales of measurement in comparison with CSLM. A first conclusion is that the CSLM estimation of the contact angles is quite good in comparison with goniometer values with the same orders of magnitude for the different system contact
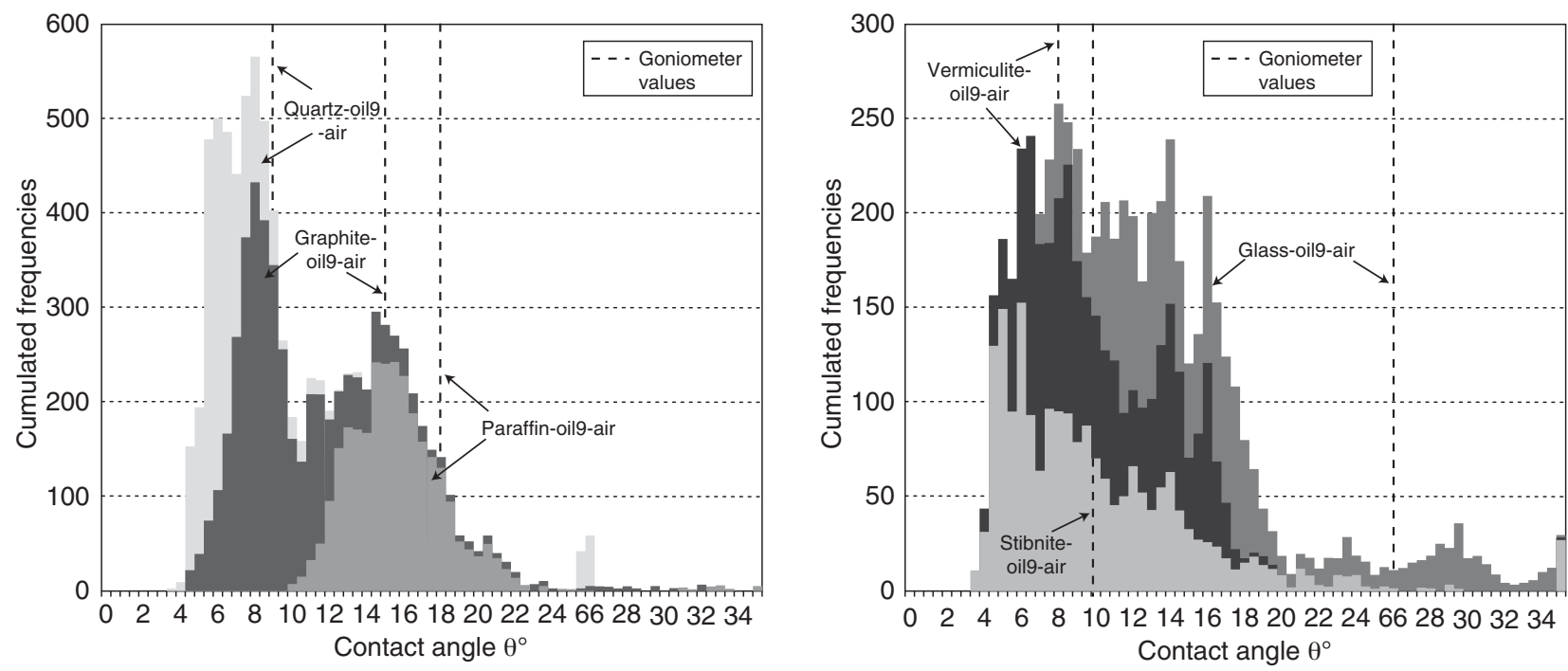

Figure 9

Frequency histograms of the contact angles calculated with the CSLM 3D models for the different three phase systems. Bars with different colors correspond to the different three phase systems. The dashed lines mark the corresponding values of contact angles determined using the goniometric method. 
angles (Fig. 8, Table 2). However, CSLM also strongly indicates that the scale of observation seems crucial to correctly discriminate the three phase contact point and that the microscopic scale of observation shows droplet irregularity and therefore a variability of the contact angle along the contact line of the three phases. The question, therefore, remains as to what is the correct scale of observation and what is the more accurate value of the contact angles.

\section{DISCUSSION}

The variability of the contact angles determined by the CSLM 3D construction is the first point to elaborate in this study. As mentioned above (Results section), the wide distribution of $\theta$ values arises from the surface construction with gOcad despite the smoothing linked to the DSI interpolation. The high or low roughness observed for the surfaces depends on the initial points used to fit the surface. These points are deduced from raw CSLM pictures after exactly the same image treatment and filtering. The difference most likely arises from the intensity of fluorescence of the oil droplets used during the recording. Natural oils are characterized by a complex chemical composition. An example of this complex chemistry is illustrated in Figure 10 where a CSLM image corresponding to the $X Y, X Z$ and $Y Z$ sections of a glass-natural oil-water system is presented. This picture shows that variations of fluorescence intensities can be detected. A

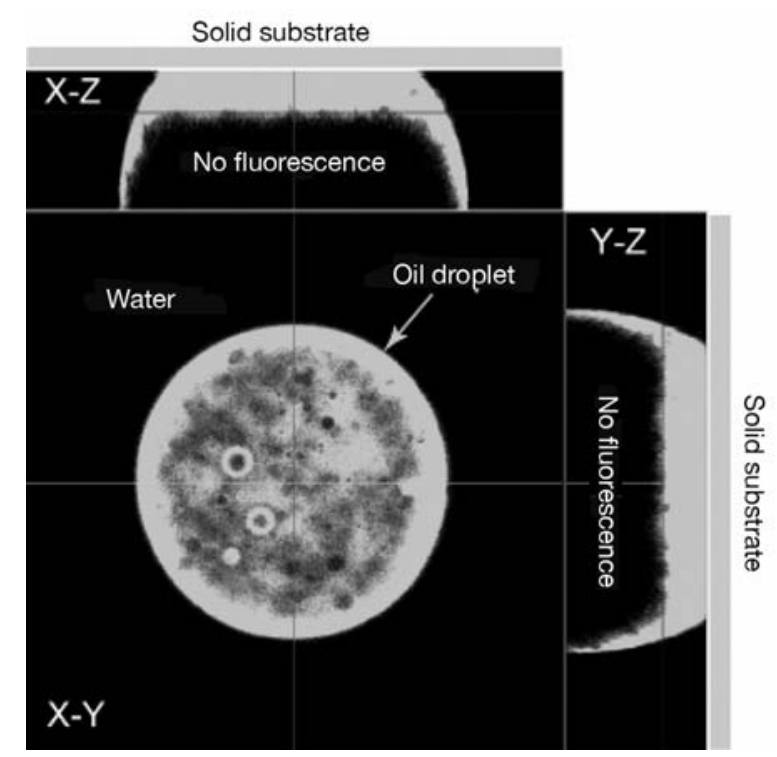

Figure 10

Water droplets inside a natural oil droplet set on a glass solid surface. Note the wide variation of fluorescence intensity inside the oil droplet. strong concentration of fluorochromes at the solid-oil and water-oil interface is evidence of the chemical heterogeneity of the oil phase but also shows that the droplet is a stable emulsion of water in oil. The water droplets appear dark on the $X Y$ section, generally surrounded by a highly fluorescent halo. This halo is assumed to be enriched in fluorochromes. The presence of numerous water droplets in oil could explain the roughness of the contour of the oil droplet. The hydrophilic character of the fluorochromes can be possibly invoked to explain the variation of fluorescence intensity. In this case, the contact angle does not correspond to the oil in itself but to a specific molecular fraction of this oil with a specific interfacial tension. CSLM is thus seen to be an efficient tool to detect fluid heterogeneity. In this case, a specific contact angle for the oil is difficult to establish with precision.

The second point is that CSLM pictures allows the definition of systematically lower or higher mean values of contact angles than the classical goniometer method (Fig. 11).

These mean values seem to be not relevant at the CSLM scale. The contact angles shown in Figure 11 show several levels and sizes of order of $\theta$. For example, in the quartz-oilair system, a first level is characterized in the vicinity of the contact zone (between 0 to $10 \mu \mathrm{m}$ ) with initial contact angle values around $6^{\circ}$. In contrast, and with higher droplet heights, $\theta$ values regularly increase and reach a value of $8^{\circ}$ corresponding approximatively to the value obtained by the goniometer analysis (Fig. 11A). This increase shows that the external perimeter of the droplet is flatter and that this zone largely spreads on the solids. The same type of observations can be made for the vermiculite-oil9-air system where again two main $\theta$ levels are defined (around $15^{\circ}$ and $7.5^{\circ}$ ). However, the higher $\theta$ value is observed between 0 and $8 \mu \mathrm{m}$ from the solid surface and then decreases with increasing droplet height. The stable $\theta$ values $\left(7^{\circ}-8^{\circ}\right)$ correspond, as previously, as nearly the goniometer value $\left(8.9^{\circ}\right)$.

In the cases presented in Figure 11, the contact angles show relatively homogeneous stable values in the vicinity of the three phase contact point with $\theta$ around $6^{\circ}$ and $15^{\circ}$ for the quartz-oil9-air and the vermiculite-oil9-air systems, respectively. These angles gradually increase (quartz-oil9-air) or decrease (vermiculite-oil9-air) to reach the goniometer values for $Z$ heights up to $10 \mu \mathrm{m}$. This implies that angles become similar between the two techniques up to a certain contact point and that this $10-15 \mu \mathrm{m}$ of height seems crucial to precisely define realistic values of $\theta$.

The morphology of the droplet is therefore more complex than a pseudo-half sphere and this complexity can be related to the solid surface quality. Practically every solid surface has spatial variations in surface chemistry and/or surface roughness. Consequently, the contact angle can vary across such a surface. This variation can distort the contact line and thus the ideal "pseudo-half sphere" droplet shape. 

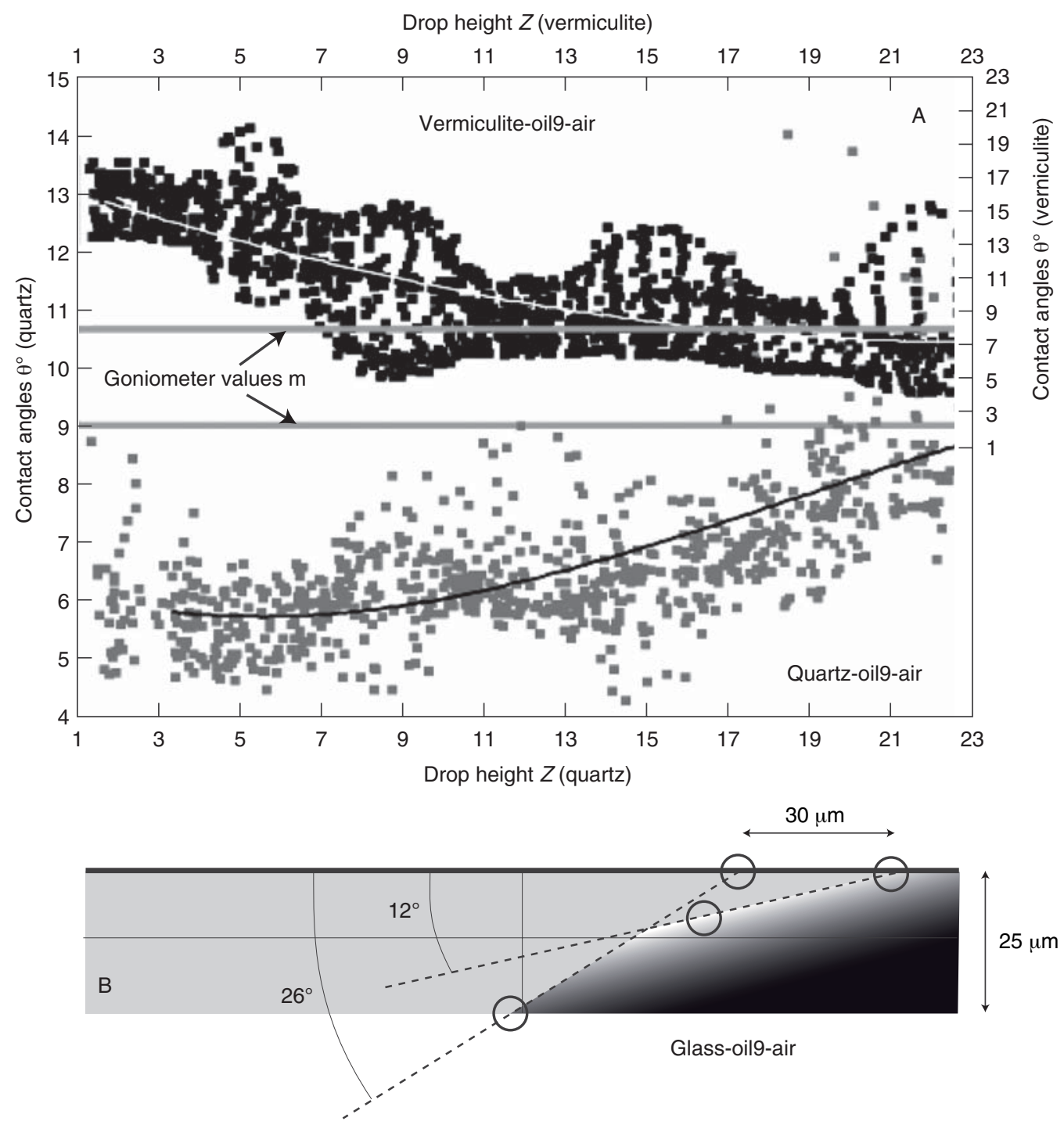

Figure 11

(A) Plot of the contact angles versus the droplet height $Z$ for two solid-oil9-air system (Vermiculite and Quartz). A continuous decrease or increase of the $\theta$ values is observed with increasing $Z$ and the goniometer values of reference are reached only for $Z$ higher than around $20 \mu \mathrm{m}$.

(B) CSLM XZ section of a droplet corresponding to the oil9-glass-system illustrating a distorted line case. Two main contact angles can be defined from two triple points spaced by $30 \mu \mathrm{m}$.

Decker et al. (1999) compared the distorted and undistorted line liquid-vapor interface near the contact line on the outside of a vertical capillary glass tube partially submerged in a water bath. These authors observed that the distortion of the interface diminished at increasing distances from the contact line and calculated the expected error on simply measuring the contact angle by placing a tangent to the fluid1-fluid2 interface at some arbitrary distance from the contact line. Indeed, the same observation can be done in the case of the glass-oil9-air system presented in Figure 11B where the $\theta$ values clearly show two trends. This picture shows that the contact point definition (large grey circle in Fig. 11B) can be moved to fit the two main slopes of the profile. Only $30 \mu \mathrm{m}$ separates the two extreme tangent drawings but this produces two angles from $12^{\circ}$ to $26^{\circ}$. The goniometer camera does not allow such precision to define a good three phase contact point. In this case, choosing the best or most appropriate value is again quite difficult. For this system, the 2D image analysis gives a mean value of $13.9^{\circ} \pm 1.3^{\circ}$. This value fits relatively well the first mode obtained at $\theta=14^{\circ}$ in Figure 9, but the goniometer value given at $26^{\circ}$ is considerably different. The goniometer value agrees quite well with the second mode of the distribution which approaches a maximal value illustrating once again that the scale effect is crucial for a precise determination of the contact angle. 


\section{CONCLUSION}

Contact angle measurement is a simple and very useful tool to evaluate the wettability and the surface energy of a material in contact with two immiscible fluids. In this paper, two main methodologies of contact angle measurement are presented and compared. The CSLM and the goniometer approaches give the same order of sizes for different solidoil-air systems. However, the 3D microscopic images of the different droplet taken by CSLM yield other important information such as, for example, the heterogeneous distribution of the fluorochromes inside the droplets and, more importantly, the problem of the scale definition of the contact angle. The gOcad model shows that the scale needed to correctly estimate the contact angle is crucial. The observed difference between the two techniques is attributed to the scale (resolution) difference. In the framework of this work, the following question could be asked: in the case of oilwater-solid system with $\theta_{\text {oil }}>90^{\circ}$, is there a real contact of oil with the solid on a nanometric scale? The answer is not probably identical for all solids and depends on the hydrophobic/hydrophilic properties of their surfaces. Such observations have consequences for the mechanism of crystal growth in oil water geological systems. The presence of a residual "protective" film of water on mineral surfaces might be suggested to explain the observation that a change in the oil chemistry in a reservoir does not totally inhibit the diagenesis (i.e. mineral formation in sediments). The CSLM technique can therefore provide a better understanding of mono and/or multiphase flow in porous or fractured rocks. Furthermore, it emphasizes the fact that, in future, we shall need to reconsider more rigorously contact angle measurements of complex natural fluids.

\section{ACKNOWLEDGMENTS}

Particular thanks are due to Mathieu PETRISSANS and Mohamed HAKKOU from LERMAB UMR (1093), UHP, Vandœuvre-lès-Nancy France for the goniometer measurements. We are also grateful to Pierrick GAUDIN from ENSG, Nancy, France for his preliminary work on the gOcad models.
This project was partly supported by the Institut National Polytechnique de Lorraine.

\section{REFERENCES}

Anderson W.G. (1987) Wettability Literature Survey-Part 4: Effects of Wettability on Capillary Pressure, J. Petrol. Technol. 38, 1125-1144.

Craig F.F. Jr. (1971) The Reservoir Engineering Aspects of Waterflooding, Society of Petroleum Engineers, Vol. 3, Dallas, USA.

Crocker M.E., Marchin L.M. (1988) Wettability and adsorption characteristics of crude-oil asphaltenes and polar fractions, $J$. Petrol.Technol. 40, 470-474.

Decker E.L., Frank B., Suo Y., Garoff S. (1999) Physics of contact angle measurement, Colloid. Surface. A 156, 177-189.

Gu Y. (2001) Drop size dependence of contact angles of oil drops on a solid surface in water, Colloid. Surface. A 181, 215-224.

Jadhunandan P.P., Morrow N.R. (1995) Effect of wettability on water-flood recovery for crude-oil/brine/rock systems, SPE Reservoir Engineering, February, pp. 40-46.

Leger L., Joanny J.F. (1992) Liquid spreading, Rep. Prog. Phys. $\mathbf{5 5}, 431-86$.

Li D. (1996) Drop size dependence of contact angles and line tensions of solid-line systems, Colloid. Surface. A 116, 1-23.

Mallet J.L. (1992) Discrete smooth interpolation in geometric modelling, Comput. Aided Design 24, 178-191.

Mallet J.L. (2002) Geomodeling, Oxford University Press, New York, $624 \mathrm{p}$.

Morrow N.R. (1991) Introduction to interfacial phenomena in oil recovery. Interfacial Phenomena in Petroleum Recovery, Surfactant science series, New York, 36, Chap. 1, pp. 1-21.

Morrow N.R., Lim L.T., Ward J.S. (1986) Effect of Crude-OilInduced Wettability Changes on Oil Recovery, SPEFE J. Feb. 1, 89-103.

Pironon J., Canals M., Dubessy J., Walgenwitz F., LaplaceBuilhe C. (1998) Volumetric reconstruction of individual oil inclusions by confocal scanning laser microscopy, Eur. J. Mineral. 10, 1143-1150.

Salathiel R.A. (1973) Oil recovery by surface film drainage in mixed-wettability rocks, J. Petrol. Technol. 25, 1216-1224.

Yang M.-W., Lin S.-Y. (2003) A method for correcting the contact angle from the $\theta / 2$ method, Colloid. Surface. A 220, 199-210.

Zhang N., Chao D.F. (2002) A new laser shadowgraphy method for measurements of dynamic contact angle and simultaneous flow visualization in a sessile drop, Opt. Laser Technol. 34, 234-248.

Final manuscript received in January 2008 Published online in September 2008

Copyright $(0) 2008$ Institut français du pétrole

Permission to make digital or hard copies of part or all of this work for personal or classroom use is granted without fee provided that copies are not made or distributed for profit or commercial advantage and that copies bear this notice and the full citation on the first page. Copyrights for components of this work owned by others than IFP must be honored. Abstracting with credit is permitted. To copy otherwise, to republish, to post on servers, or to redistribute to lists, requires prior specific permission and/or a fee: Request permission from Documentation, Institut français du pétrole, fax. +33147527078 , or revueogst@ifp.fr. 